\title{
The Role of Contractile Reserve by Stress Test Echocardiography for Predicting Cardiac Resynchronization Therapy Responder: Systematic Review and Meta-analysis
}

\author{
Achmad Lefi ${ }^{1 *}$, Ivana Purnama Dewi ${ }^{1,3}$, Kristin Purnama Dewi ${ }^{2}$, Eka Prasetya Budi Mulia ${ }^{1}$, Agus Subagjo $^{1}$, \\ Budi Baktijasa Dharmadjati ${ }^{1}$ \\ ${ }^{1}$ Department of Cardiology and Vascular Medicine, Faculty of Medicine, Airlangga University - Dr. Soetomo General Hospital, \\ Surabaya, Indonesia, ${ }^{2}$ Department of Pulmonology and Respiratory Medicine, Faculty of Medicine, Airlangga University - \\ Dr. Soetomo General Hospital, Surabaya, Indonesia, ${ }^{3}$ Faculty of Medicine, Duta Wacana Christian University, Yogyakarta, Indonesia
}

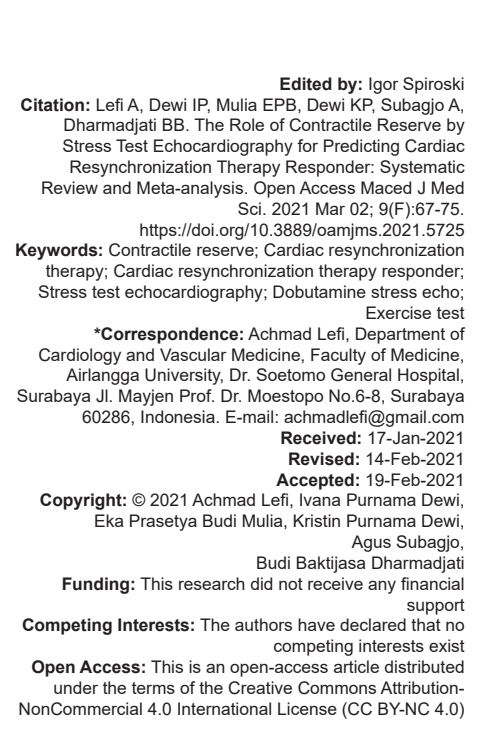

\section{Abstract}

BACKGROUND: Up to one-third of patients who received resynchronization devices do not experience full benefit of cardiac resynchronization therapy (CRT). Echocardiography plays an important role in heart failure patients treated with CRT. Contractile reserved is a strong prognostic factor to predict positive response to CRT (CRT responder).

AIM: We perform a systematic review and meta-analysis of published data to assess the relationship between contractile reserve (CR) and response to CRT.

METHODS: We conduct a systematic search from major medical databases on all clinical trials published up to June 2020, written in English, full-text availability, and human subject. We used Newcastle-Ottawa Scale to measure the quality of evidence. We employed the Mantel-Haenszel random-effects meta-analysis of using RevMan 5.4.

RESULTS: We identified 17 studies involving 1399 patients. The type of stress was either dobutamine $(n=15)$ or exercise $(n=2)$. The presence of $C R$ was associated with a higher chance of CRT responder (odds ratio [OR] $7.68,95 \%$ confidence interval $[\mathrm{Cl}] 4.27-13.82, \mathrm{p}<0.001)$ using a random-effects model. The ORs slightly differed when studies were analyzed separately based on the stress test type and definition of CR. CR, assessed with dobutamine stress echocardiography, defined as an increase in left ventricular systolic function (OR 5.10, 95\% $\mathrm{Cl} 2.29-11.32$, p < 0.00001) was numerically lower than defined as increased LV contractility (OR 6.86, 95\% Cl $3.36-12.88, p<0.00001)$. The presence of CR assessed with exercise stress test is associated with higher chance of CRT responder (OR 49.11, 95\% Cl 15.04-160.36, $\mathrm{p}<0.00001)$. From our meta-analysis, we found non-ischemic has better respond to CRT compare to patient with ischemic etiology (OR 0.41; 95\% Cl 0.31-0.55, p < 0.01)

CONCLUSION: The presence of CR during stress test echocardiography with either dobutamine or exercise stress test is associated with a higher chance of CRT responder.

\section{Introduction}

Congestive heart failure is a cardiovascular disease with an increasing incidence. Cardiac resynchronization therapy (CRT) is an adjunct therapy in the management of end-stage heart failure that is resistant to medical therapy. Studies with large sample sizes have reported benefits of CRT in patients with severe heart failure (New York Heart Association/NYHA class III/IV), decreased left ventricular systolic function (LVEF $\leq 35 \%$ ); and wide QRS complex (>120 ms) [1], [2]. The positive benefits of CRT include improvement of clinical complaints, stroke volume (SV), LVEF, mitral regurgitation, left ventricle (LV) remodeling, and survival rate.

One of the issues in CRT therapy is the identification of prospective patients who will get benefit from CRT implantation. The duration of the QRS complex is still the only parameter indicative of CRT implantation in patients with severe symptomatic heart failure with a decreased ejection fraction [3]. Approximately $20-30 \%$ of CRT patients, selected based on QRS complex duration criteria, are non-CRT responder [4]. Other factors, such as myocardial viability, determine the placement of the CRT lead. Lead position in the scar tissue is a determinant of poor response to CRT [5]. LV pacing becomes less efficient because fibrotic tissue can modify myocardial contractility and electrical conduction of the heart [6]. Myocardial viability can be assessed by several non-invasive modalities, such as nuclear imaging, dobutamine stress echocardiography (DSE), and magnetic resonance imaging.

Contractile reserve (CR) is one of global myocardial contractility markers that are related to myocardial viability. CR assessment correlates with the extent of fibrosis and is a modality with high sensitivity and specificity in assessing the response to CRT 
implantation [7]. CR is also a simple parameter that is easy to do in daily practice. Two meta-analysis about CR in predicting CRT responder were conducted by Ciampi et al., and Kloosterman et al., in 2017 [8], [9]. However, from the two meta-analyzes, the study results were quite heterogeneous. We performed an update metaanalysis by adding three studies with a quite large total sample from three studies. We also evaluate ischemic etiology as factors that related to CRT responder.

$\mathrm{CR}$ is a parameter measured by visual myocardium motion and/or LVEF changes by stress echocardiography (SE). The stimulus for SE can be done in 2 ways: Dobutamine or exercise. The aim of this article is to provide a critical assessment of existing studies of CR assessment in predicting CRT responder, provide clinically useful reference values in patients with heart failure, and test its accuracy through meta-analysis.

\section{Methods}

This study was conducted in a series of steps in accordance with the Preferred Reporting Items for Systematic Reviews and Meta-Analyzes guidelines, including searching for articles, assessing the quality of each article, extracting and analyzing data, as well as summary and interpretation of findings [10].

\section{Literature search}

We searched all articles assessing CR as a predictor in CRT candidate patients. The search was conducted systematically on various major medical databases (PubMed, Clinical Key, medRxiv, MEDLINE, Cochrane Library, and EMBASE) using keywords: "Contractile reserve," "myocardial viability," "cardiac resynchronization therapy," "CRT," "stress echocardiography," "dobutamine," and "DSE" in the title, abstract, and medical subject heading. Search parameters were limited to clinical trials, fully published studies or studies in progress if preliminary results were published, articles in English, and human studies. Lists of references from the literature matching the inclusion requirements have also been screened manually to find more relevant studies.

\section{Study selection and outcome}

We included all studies in which $\mathrm{CR}$ was assessed by DSE and/or exercise in response to CRT. The inclusion criteria are; (1) Human studies with adult sample with indications of CRT implantation according to heart failure guideline criteria (NYHA class III and/ or IV, LVEF <35\%, and QRS complex $\geq 120 \mathrm{~ms}$ ); (2)
CRT and SE were performed in the same patient population; (3) availability of CR parameter data before and after CRT implantation; (4) clear definition of response to CRT either by clinical or echocardiography assessment; and (5) followed up for at least 6 months post CRT implantation. The exclusion criteria were (1) focus on specific populations (CRT super-responders, post-implantable cardioverter defibrillator implantation population, and patients with aortic stenosis or septal flash); (2) case report study, case series, review, editorial, or conference paper; and (3) missing required data.

Before full-text retrieval, three investigators independently screened and analyzed titles and abstracts. For final inclusion, two authors analyzed the full papers that theoretically met the inclusion/exclusion criteria for final inclusion.

The primary outcome was to predict CRT responder based on $C R$ evaluation using $S E$. CR is defined as one or a combination of echocardiographic parameters: Increase LVEF and/or decrease myocardial wall contractility score (wall motion score index [WMSI]). The WMSI score is assessed based on a 16 or 17 segment model of LV recommended by the American Society of Echocardiography (ASE). Segments were scored from normal, hypokinetic, akinetic, and dyskinetic. CRT responses were assessed after CRT implantation and compared to preliminary data before CRT implantation. Positive response to CRT (CRT responder) was defined as both clinically (improvement of NYHA class, electrocardiography parameters, and pacemaker data) and by echocardiography parameters (increase LVEF and decrease left ventricular endsystolic volume).

\section{Quality assessment and publication bias}

Two investigators assessed the methodological quality of each included article using Newcastle-Ottawa Scale (NOS) for cohort studies [11]. The evaluation of NOS was carried out by considering several factors: Study selection, comparison, and outcomes. Publication bias was assessed using visual inspection of funnel plots and Harbord's regression test.

\section{Data extraction and analysis}

Two investigators extracted the data, including authors, year of publication, location, sample size, type of stress, CR criteria, duration of follow-up, and CRT responder criteria. All extracted data were recorded with a dedicated form on an Excel spreadsheet.

Meta-analysis with Mantel-Haenszel fixedeffects model using Review Manager (RevMan v5.3 2014) and Stata v.16 was conducted to assess the relationship between $\mathrm{CR}$ and response to CRT. The outcome assessment was measured using the odds 
ratio (OR). We performed a subgroup analysis based on types of SE and definition of CR. The sensitivity analysis was performed by excluding studies assessed as having a high risk of bias. Evaluation of heterogeneity between and heterogeneity in the study was carried out using the chi-square test and $\mathrm{I}^{2}$. Random-effect model analysis was performed if the $\mathrm{I}^{2}$ statistic showed more than $50 \%$ heterogeneity. Continuous data are presented as mean \pm SD and dichotomous variables are presented as percentages (\%). The statistical significance was stated if the $p$-value was $<0.05$. Restricted maximum likelihood random-effects metaregression was performed for age, sex, LVEF, QRS duration, follow-up, and ischemic etiology.

\section{Results}

The initial systematic search returned 752 articles, and 37 relevant articles were added from the main article reference list, for a total of 789 articles. We got 80 full-text studies, and 47 duplicates were excluded, leaving 33 articles. Furthermore, screening the title and abstract according to the inclusion and exclusion criteria eliminated 7 articles. A thorough reading of the full articles of the most recent number of papers resulted in an exclusion of 9 articles, due to follow-up duration $<6$ months, specific study population, unavailability of stress test intervention data, and unclear definition of CR (Figure 1).

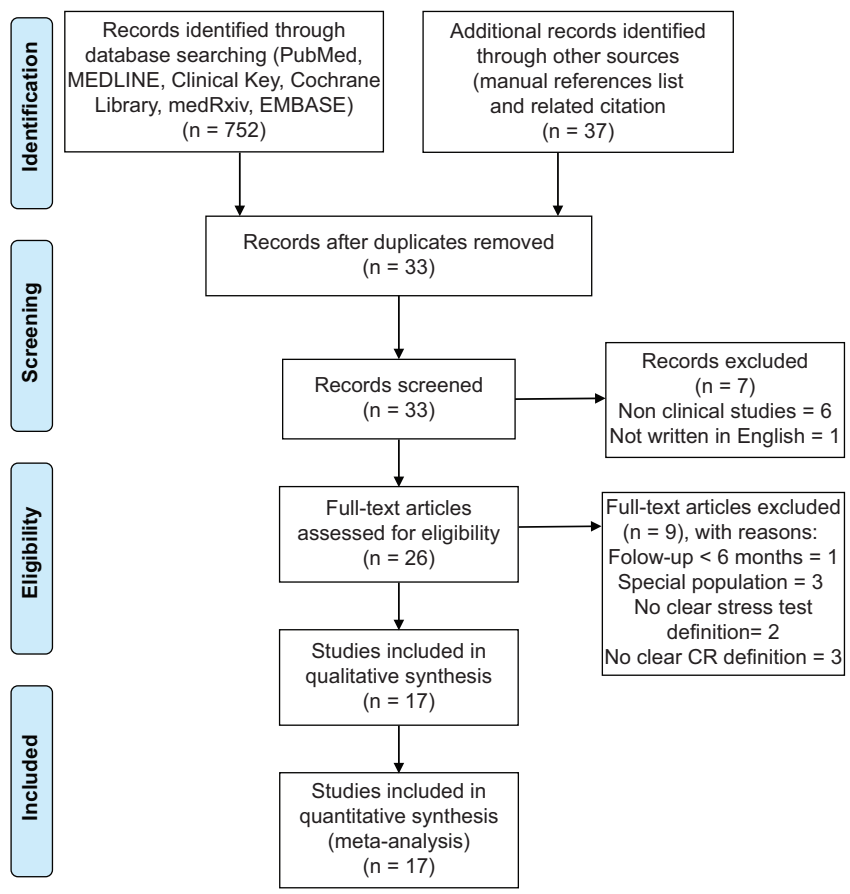

Figure 1: Literature search flow chart based on Preferred Reporting Items for Systematic Reviews and Meta-Analyses

Seventeen studies with 1399 patients (mean age $66 \pm 10$ years and $74 \%$ men) were included in this meta-analysis. Baseline of the included studies, populations are presented in Table 1. Ischemic etiology was present in $53 \%$ of patients, with mean LVEF at rest of $26 \pm 6 \%$, and a QRS complex duration of $157 \pm 24$ ms. Fifteen studies assessed CR using the DSE and two studies using exercise. Low-dose dobutamine (up to $10 \mathrm{mcg}$ ) was obtained in four studies, medium dose (up to $20 \mathrm{mcg}$ ) in nine studies and high dose protocols (up to $40 \mathrm{mcg}$ ) were obtained in two studies. The mean follow-up duration was 12 months (Table 1). CR defined by increasing in LVEF was found in 9 studies, whereas CR defined by decreasing in WMSI after a SE stimulus was found in 8 studies. One study define CR as either increasing in LVEF or decreasing in WMSI [12], [13], [14], [15], [16], [17], [18], [19], [20], [21], [22], [23], [24], [25], [26], [27], [28].

From a mean follow-up of 12 months, out of a total of 1399 patients, only 1356 patients were included by the end of the study. This is due to some patients died during follow-up or incomplete paired data at the end of the study. Total 935 patients (68\%) were CRT responders based on clinical and echocardiographic criteria. Positive CR was obtained in 908 patients (67\%). We found a higher patient prevalence of CRT responder with positive CR $(735 / 935 ; 79 \%)$. Positive predictive value was $81 \%$ and negative predictive value was $56 \%$ (Table 2 ).

Although all articles were published in peerreviewed journals, we assessed methodological quality using NOS (Table 3). Overall, all included studies have fair and good methodological quality. Visual inspection on funnel plot showed symmetry, which indicates no publication bias of all the included studies (Figure 2). Sub-group analysis based on dobutamine or exercise SE also showed symmetry in both sub-groups. Harbord's regression test showed no publication bias in overall included studied ( $p=0.105)$ and dobutamine SE subgroup $(p=0.116)$. We did not perform Harbord's regression test on exercise SE because there were $<10$ studies.

The primary outcome of this meta-analysis was a response to CRT based on baseline CR. Seventeen studies involving 1365 patients reported CR and non-CR on CRT responders (Figure 3). Contractile reserved was obtained in $67 \%(908 / 1365)$ of CRT responder. $\mathrm{CR}$ group was associated with a higher CRT responder compared with the non-CR group (OR 7.68; 95\% confidence interval [Cl] 4.27-13.82; $\mathrm{p}<0.001)$. There was significant heterogeneity $\left(\mathrm{I}^{2}=73 \%\right.$; $\left.\mathrm{p}<0.001\right)$ for the entire population.

Fifteen studies involving 1250 patients reported $\mathrm{CR}$ and non-CR assessed by DSE on response to CRT (Figure 4). CR was found in $66 \%$ (831/1250) of patients who responded to CRT. CR group was associated with a higher CRT responder compared with the non-CR group (OR 5.90; 95\% Cl 3.37-10.34; $p<0.001)$. There was significant heterogeneity $\left(\mathrm{I}^{2}=68 \% ; \mathrm{p}<0.001\right)$. We also performed a subgroup analysis based on CR definition: 
Table 1: Baseline characteristics of the studies

\begin{tabular}{|c|c|c|c|c|c|c|c|c|c|c|c|c|c|}
\hline Author, Years & Country & $\mathrm{n}$ & $\begin{array}{l}\text { Male } \\
(\%)\end{array}$ & $\begin{array}{l}\text { Age } \\
\text { (years) }\end{array}$ & $\begin{array}{l}\text { LVEF } \\
(\%)\end{array}$ & $\begin{array}{l}\text { QRS } \\
\text { duration }\end{array}$ & $\begin{array}{l}\text { Ischemic } \\
(\%)\end{array}$ & $\begin{array}{l}\text { NYHA } \\
\text { class III/IV } \\
(\%)\end{array}$ & Stress Type & $\begin{array}{l}\text { Dobutamine } \\
\text { dose }(\mathrm{mg})\end{array}$ & $\begin{array}{l}\text { CRT Response } \\
\text { (Responder) }\end{array}$ & $\begin{array}{l}\text { Contractile } \\
\text { reserved }\end{array}$ & $\begin{array}{l}\text { Follow-up } \\
\text { (months) }\end{array}$ \\
\hline $\begin{array}{l}\text { Da Costa, } \\
2005 \text { [9] }\end{array}$ & France & 67 & 84 & $64 \pm 10$ & $26 \pm 5$ & $154 \pm 30$ & 34 & $60 / 40$ & Dobutamine & 10 & $\begin{array}{l}\text { Clinical (NYHA, ECG, } \\
\text { Pacemaker data) }\end{array}$ & $\begin{array}{l}\text { Increase } \\
E F>1.25 \text { fold }\end{array}$ & 20 \\
\hline $\begin{array}{l}\text { Ypenburg, } \\
2007[10]\end{array}$ & Netherlands & 31 & 87 & $71 \pm 10$ & $26 \pm 7$ & $191 \pm 28$ & 65 & $90 / 3$ & Dobutamine & 10 & Decrease LVESV $\geq 15 \%$ & $\begin{array}{l}\text { Increase } \\
E F>7.5 \%\end{array}$ & 6 \\
\hline $\begin{array}{l}\text { Lim, } \\
2007 \text { [11] }\end{array}$ & France & 19 & 74 & $64 \pm 13$ & $27 \pm 8$ & $154 \pm 25$ & 47 & $79 / 21$ & dobutamine & 10 & Decrease LVESV & $\begin{array}{l}\text { Decrease } \\
\text { WMSI } \geq 0.25\end{array}$ & 6 \\
\hline $\begin{array}{l}\text { Tuccillo, } \\
2008 \text { [12] }\end{array}$ & Italy & 42 & 76 & $66 \pm 9$ & $27 \pm 7$ & $144 \pm 23$ & 43 & $88 / 12$ & Dobutamine & 20 & Decrease LVESV $\geq 15 \%$ & $\begin{array}{l}\text { Increase } \\
E F>25 \%\end{array}$ & 6 \\
\hline $\begin{array}{l}\text { Rocchi, } \\
2008 \text { [13] }\end{array}$ & Italy & 64 & 75 & $64 \pm 11$ & $29 \pm 5$ & $154 \pm 25$ & 31 & $95 / 5$ & Exercise & - & $\begin{array}{l}\text { Decrease LVESV } \geq 15 \% \\
\text { and/or increase } \\
\text { LVEF }>5 \% \text { (absolute value) }\end{array}$ & $\begin{array}{l}\text { Decrease } \\
\text { WMSI } \geq 0.25\end{array}$ & 6 \\
\hline $\begin{array}{l}\text { Ciampi, } \\
2009[14]\end{array}$ & Italy & 69 & 71 & $70 \pm 8$ & $27 \pm 6$ & $150 \pm 27$ & 55 & $83 / 17$ & Dobutamine & 40 & Decrease LVESV $\geq 15 \%$ & $\begin{array}{l}\text { Decrease } \\
\text { WMSI } \geq 0.20\end{array}$ & 11 \\
\hline $\begin{array}{l}\text { Lancelloti, } \\
2009 \text { [15] }\end{array}$ & Belgium & 51 & 63 & $70 \pm 9$ & $27 \pm 5$ & $161 \pm 25$ & 67 & $3.1^{*}$ & Exercise & - & Decrease LVESV $\geq 15 \%$ & $\begin{array}{l}\text { Increase } \\
E F>6.5 \%\end{array}$ & 6 \\
\hline $\begin{array}{l}\text { Muto, } \\
2010[16]\end{array}$ & Italy & 231 & 72 & $67 \pm 10$ & $27 \pm 6$ & $149 \pm 24$ & 43 & $94 / 6$ & Dobutamine & 20 & Decrease LVESV $\geq 10 \%$ & $\begin{array}{l}\text { Increase } \\
E F>5 \text { points }\end{array}$ & 6 \\
\hline $\begin{array}{l}\text { Senechal, } \\
2010[17]\end{array}$ & Belgium & 49 & 69 & $66 \pm 12$ & $19 \pm 7$ & $164 \pm 30$ & 69 & $67 / 33$ & Dobutamine & 20 & Decrease LVESV $\geq 15 \%$ & $\begin{array}{l}\text { Decrease } \\
\text { WMSI } \geq 0.25\end{array}$ & 6 \\
\hline $\begin{array}{l}\text { Altman, } \\
2011[18]\end{array}$ & USA & 31 & 74 & $68 \pm 12$ & $28 \pm 6$ & $158 \pm 22$ & 65 & $90 / 10$ & Dobutamine & 10 & Decrease LVESV $\geq 15 \%$ & $\begin{array}{l}\text { Increase } \\
E F>20 \% \\
\text { (relative) }\end{array}$ & 24 \\
\hline $\begin{array}{l}\text { Gasparini, } \\
2011 \text { [19] }\end{array}$ & Italy & 221 & 70 & $67 \pm 10$ & $27 \pm 6$ & $150 \pm 25$ & 43 & $95 / 5$ & Dobutamine & 20 & Decrease LVESV>10\% & $\begin{array}{l}\text { Increase } \\
E F>5 \text { points }\end{array}$ & $15 \pm 5$ \\
\hline $\begin{array}{l}\text { Chaudhry, } \\
2011 \text { [20] }\end{array}$ & Netherlands & 54 & 63 & $69 \pm 11$ & $18 \pm 5$ & $147 \pm 20$ & 59 & $3.2^{*}$ & Dobutamine & 20 & Increase LVEF $>5$ points & $\begin{array}{l}\text { Decrease } \\
\text { WMSI } \geq 0.31\end{array}$ & 7 \\
\hline $\begin{array}{l}\text { Pugllese, } \\
2012[21]\end{array}$ & Italy & 104 & 64 & $71 \pm 5$ & $27 \pm 4$ & $142 \pm 11$ & 87 & $3.2^{*}$ & Dobutamine & 20 & $\begin{array}{l}\text { Decrease LVESV } \geq 15 \% \\
\text { and/or increase LVEF }>5 \%\end{array}$ & $\begin{array}{l}\text { Decrease } \\
\text { WMSI } \geq 0.20\end{array}$ & 6 \\
\hline $\begin{array}{l}\text { Stankovic, } \\
2013[22]\end{array}$ & Norway & 58 & 78 & $63 \pm 10$ & $26 \pm 6$ & $175 \pm 25$ & 47 & $3.1^{*}$ & Dobutamine & 20 & Decrease LVESV>10\% & $\begin{array}{l}\text { Increase } \\
E F>5 \%\end{array}$ & $41 \pm 13$ \\
\hline $\begin{array}{l}\text { Mizia-Stec, } \\
2013 \text { [23] }\end{array}$ & Poland & 129 & 76 & $62 \pm 9$ & $25 \pm 6$ & $164 \pm 24$ & 48 & $90 / 10$ & Dobutamine & 20 & Decrease LVESV $\geq 15 \%$ & $\begin{array}{l}\text { Decrease } \\
\text { WMSI } \geq 0.20\end{array}$ & 12 \\
\hline $\begin{array}{l}\text { Murin, } \\
2014[24]\end{array}$ & Slovak Rep & 52 & 75 & $62 \pm 11$ & $26 \pm 7$ & $150 \pm 24$ & 48 & $100 / 0$ & Dobutamine & 40 & $\begin{array}{l}\text { Decrease LVESV } \geq 15 \% \\
\text { and/or increase LVEF }>5 \%\end{array}$ & $\begin{array}{l}\text { Increase } \\
E F>7 \%\end{array}$ & 6 \\
\hline $\begin{array}{l}\text { Plonska-Gosciniak, } \\
2016 \text { [25] }\end{array}$ & Poland & 127 & 79 & $63 \pm 9$ & $25 \pm 6$ & $163 \pm 23$ & 48 & $76 / 9$ & Dobutamine & 20 & Decrease LVESV>10\% & $\begin{array}{l}\text { Increase } \\
\mathrm{EF}>20 \% \text { or } \\
\text { decrease } \\
\text { WMSI }\end{array}$ & 12 \\
\hline
\end{tabular}
index. LVEF: Left ventricular systolic function.

Table 2: CR with SE in patients with CRT responder and non-CRT responder

\begin{tabular}{|c|c|c|c|c|c|c|c|c|c|c|c|c|}
\hline Author, Years & $\mathrm{CRT}+, \mathrm{CR}+$ & $\%$ & CRT+, CR - & $\%$ & CRT-, CR + & $\%$ & CRT-, CR - & $\%$ & CRT+ & $\%$ & $\mathrm{CR}+$ & $\%$ \\
\hline Da Costa, 2005 [10] & 28 & 42 & 19 & 28 & 6 & 9 & 14 & 21 & 47 & 70 & 34 & 51 \\
\hline Ypenburg, 2007 [11] & 13 & 42 & 4 & 13 & 2 & 6 & 12 & 39 & 17 & 55 & 15 & 48 \\
\hline Lim, 2007 [12] & 10 & 53 & 3 & 16 & 0 & 0 & 6 & 31 & 13 & 68 & 10 & 77 \\
\hline Tuccillo, 2008 [13] & 25 & 59 & 0 & 0 & 2 & 5 & 15 & 36 & 25 & 60 & 27 & 64 \\
\hline Rocchi, 2008 [14] & 41 & 64 & 2 & 3 & 5 & 8 & 16 & 25 & 43 & 67 & 46 & 72 \\
\hline Ciampi, 2009 [15] & 29 & 49 & 5 & 7 & 13 & 22 & 12 & 20 & 34 & 59 & 42 & 71 \\
\hline Lancelloti, 2009 [16] & 27 & 53 & 3 & 6 & 4 & 8 & 17 & 33 & 30 & 59 & 31 & 61 \\
\hline Muto, 2010 [17] & 145 & 63 & 25 & 11 & 40 & 17 & 21 & 9 & 170 & 74 & 185 & 80 \\
\hline Senechal, 2010 [18] & 30 & 61 & 1 & 2 & 7 & 14 & 11 & 23 & 31 & 63 & 37 & 63 \\
\hline Altman, 2011 [19] & 11 & 35 & 3 & 10 & 9 & 29 & 8 & 26 & 14 & 45 & 20 & 65 \\
\hline Gasparini, 2011 [20] & 144 & 70 & 16 & 8 & 22 & 11 & 22 & 11 & 160 & 78 & 166 & 81 \\
\hline Chaudhry, 2011 [21] & 29 & 54 & 2 & 4 & 13 & 24 & 10 & 18 & 31 & 57 & 42 & 78 \\
\hline Pugllese, 2012 [22] & 37 & 38 & 19 & 19 & 14 & 14 & 28 & 29 & 56 & 57 & 51 & 49 \\
\hline Stankovic, 2013 [23] & 15 & 26 & 24 & 41 & 10 & 17 & 9 & 16 & 39 & 67 & 25 & 43 \\
\hline Mizia-Stec, 2013 [24] & 62 & 48 & 42 & 33 & 5 & 4 & 20 & 15 & 104 & 81 & 67 & 52 \\
\hline Murin, 2014 [25] & 22 & 43 & 6 & 12 & 3 & 6 & 20 & 39 & 28 & 55 & 25 & 49 \\
\hline Plonska-Gosciniak, 2016 [26] & 67 & 53 & 26 & 20 & 18 & 14 & 16 & 13 & 93 & 73 & 85 & 67 \\
\hline
\end{tabular}

CR: Contractile reserve; CRT: Cardiac resynchronization therapy; SE: Stress echocardiography.

Table 3: NOS quality assessment

\begin{tabular}{|c|c|c|c|}
\hline Author, Years & Selection & Comparability & Outcome \\
\hline Da Costa, 2005 [10] & 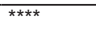 & * & $\star \star \star *$ \\
\hline Ypenburg, 2007 [11] & $\star \star \star \star *$ & * & *** \\
\hline Lim, 2007 [12] & 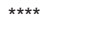 & * & $\star \star * *$ \\
\hline Tuccillo, 2008 [13] & **** & * & ** \\
\hline Rocchi, 2008 [14] & 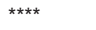 & * & *** \\
\hline Ciampi, 2009 [15] & 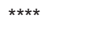 & * & $\star \star \star *$ \\
\hline Lancelloti, 2009 [16] & $* * * *$ & * & *** \\
\hline Muto, $2010[17]$ & 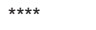 & * & ** \\
\hline Senechal, 2010 [18] & **** & * & *** \\
\hline Altman, 2011 [19] & **** & * & ** \\
\hline Gasparini, 2011 [20] & 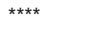 & * & *** \\
\hline Chaudhry, 2011 [21] & $* \star \star * *$ & * & $* * *$ \\
\hline Pugllese, 2012 [22] & $\star * * *$ & * & $* \star *$ \\
\hline Stankovic, 2013 [23] & $\star \star \star \star *$ & * & ** \\
\hline Mizia-Stec, 2013 [24] & 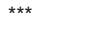 & * & ** \\
\hline Murin, 2014 [25] & **** & * & *** \\
\hline Plonska-Gosciniak, 2016 [26] & 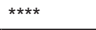 & * & $\star \star \star *$ \\
\hline
\end{tabular}

Increased LVEF and decreased WMSI score. Subgroup analysis of nine studies included 842 patients which CR was assessed based on increased LVEF showed a similar result: CR group was associated with a higher CRT responder compared with non-CR group (OR 5.10; 95\% Cl 2.29-11.32; $p<0.001$ ) with significant heterogeneity $\left(I^{2}=77 \% ; p<0.001\right)$. Six studies included 408 patients, which $\mathrm{CR}$ was assessed based on decreased WMSI scores also showed a similar result: CR group was associated with a higher CRT responder compared with non-CR group (OR 5.90; 95\% Cl 3.37-10.34; $p<0.001$ ) without significant heterogeneity $\left(I^{2}=21 \% ; p=0.27\right)$. 


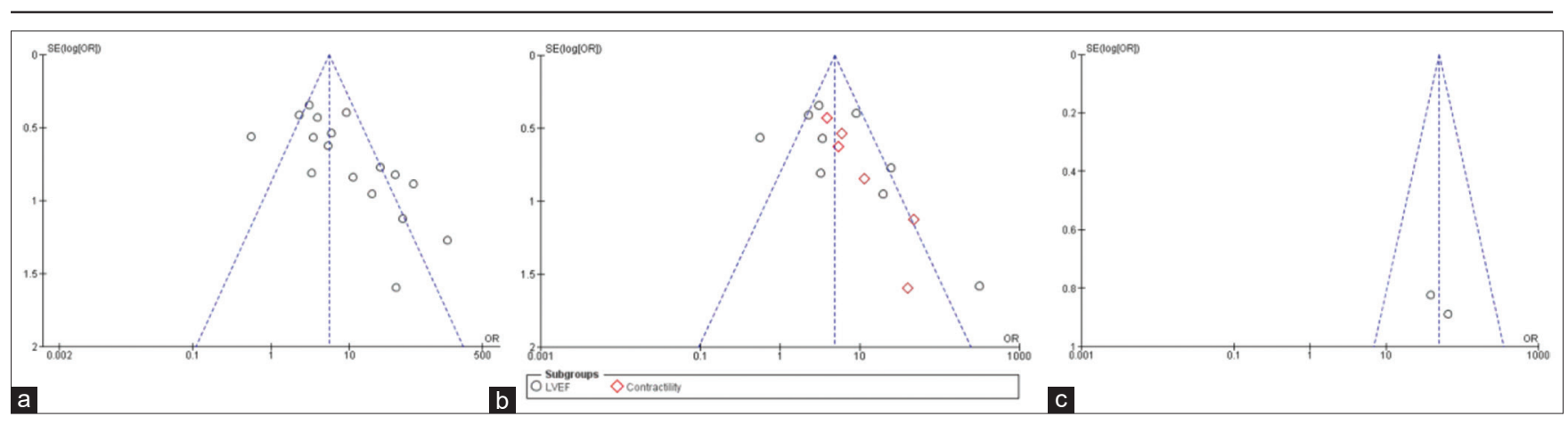

Figure 2: Funnel plots of study outcome showed no publication bias for (a) all included studies ( $p=0.105)$; (b) dobutamine stress echocardiography $(p=0.116) ;(c)$ exercise stress echocardiography

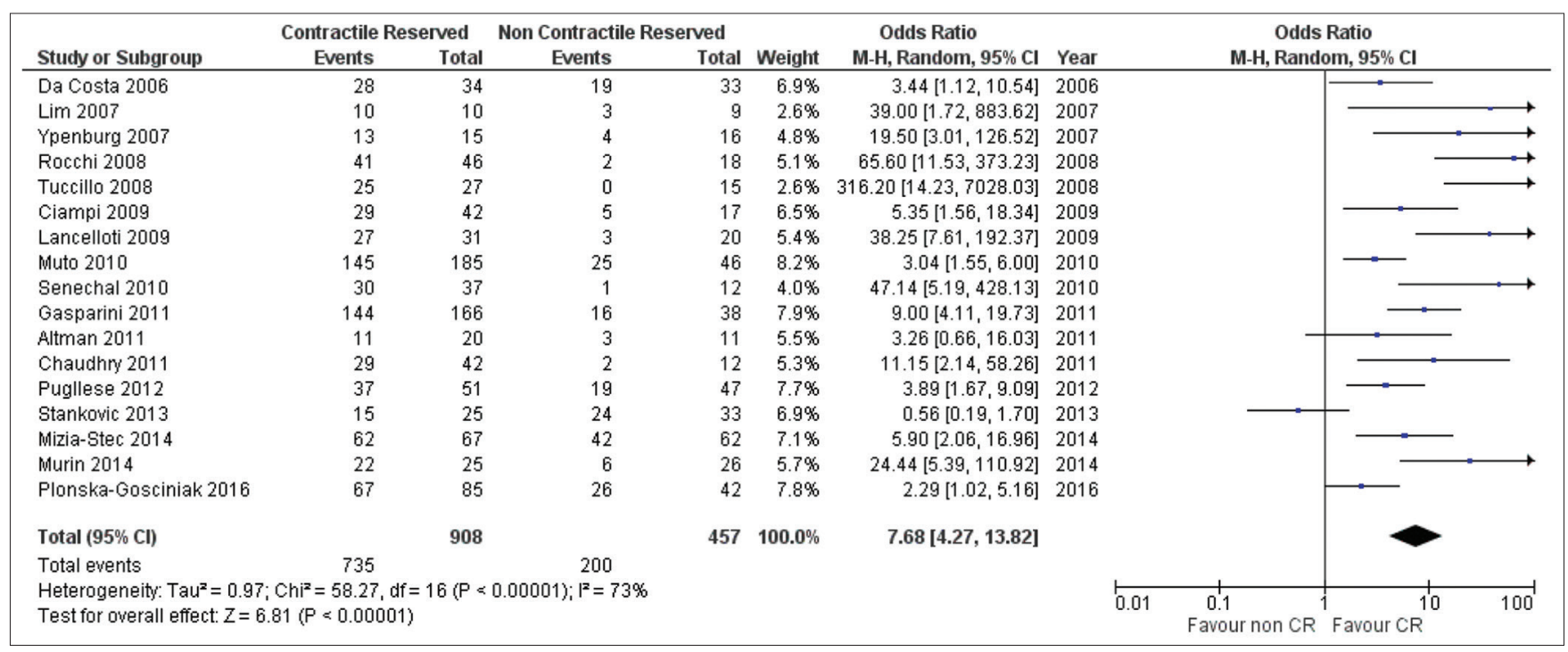

Figure 3: Forest plots of cardiac resynchronization therapy responder based on contractile reserve in all included studies

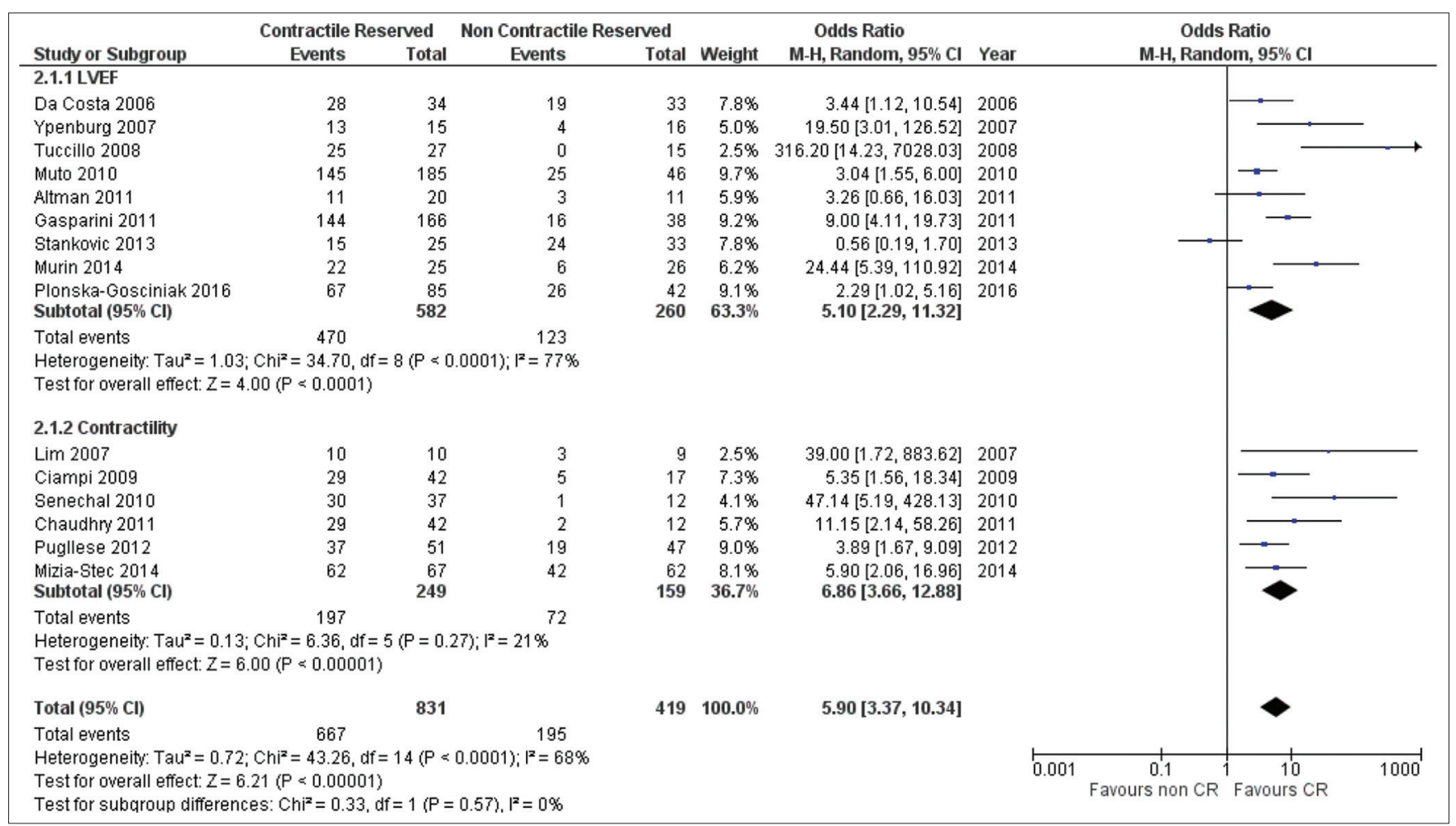

Figure 4: Forest plots of cardiac resynchronization therapy responder based on contractile reserve assessed by dobutamine stress echocardiography 
Two studies involving 115 patients reported CR and non-CR assessed by exercise SE on CRT responder (Figure 5). CR was found in $67 \%$ (77/115) of patients who responded to CRT. CR group was associated with a higher CRT responder compared to non-CR group (OR 49.11; 95\% Cl 15.04-60.36; $p<0.001$ ) without any significant heterogeneity $\left(I^{2}=0 \% ; p=0.66\right)$.

Random-effects meta-regression analysis showed that the association between $\mathrm{CR}$ and CRT responder was not significantly affected by age $(p=0.451)$, sex $(p=0.612)$, LVEF $(p=0.978)$, QRS duration $(p=0.968)$, follow-up $(p=0.259)$, and ischemic etiology $(p=0.821)$.

We also performed a group analysis of 13 studies that included ischemic etiology with CRT responder (Figure 6). The overall OR benefit of CRT in ischemic etiology was $0.41(95 \% \mathrm{Cl} 0.31-0.55$, $p<0.01)$ without significant heterogeneity $\left(I^{2}=32 \%\right.$; $\mathrm{p}<0.15)$.

\section{Discussion}

CRT has shown considerable potential to improve outcomes in patients with severe chronic heart failure. Large studies showed that CRT could improve symptoms of heart failure, LV function, exercise capacity, and reduce morbidity and mortality [29], [30]. Several previous studies have also demonstrated the efficiency of mechanical resynchronization in ischemic and nonischemic cardiomyopathy. However, $40 \%$ of patients who underwent CRT implantation did not respond well enough so that other criteria were needed in selecting CRT patient candidates [29], [30]. Myocardial viability is one of the current factors in clinical practice. Myocardial viability can be measured by SE by looking at the presence of $C R$. The presence of $C R$ increases the possibility of a positive CRT response and could be one of the considerations in selection of CRT candidates. SE defined groups with a better prognosis of enhancing clinical and functional conditions after CRT implantation in the $\mathrm{CR}$ evaluation [8].

In 2016, ESC guidelines regarding the diagnosis and management of heart failure, clinical symptoms, ejection fraction, and QRS complex duration are still major criteria for identifying heart failure patients as CRT candidates [3]. ESC guidelines suggest an exercise or pharmacological SE for the assessment of myocardial viability and/or myocardial ischemia but do not mention SE to identify CRT responder [3].

Mechanical dyssynchrony has been described as the regional contraction discrepancy or an uncoordinated, unequaled regional myocardial motion [31]. Left bundle branch block (LBBB) may trigger

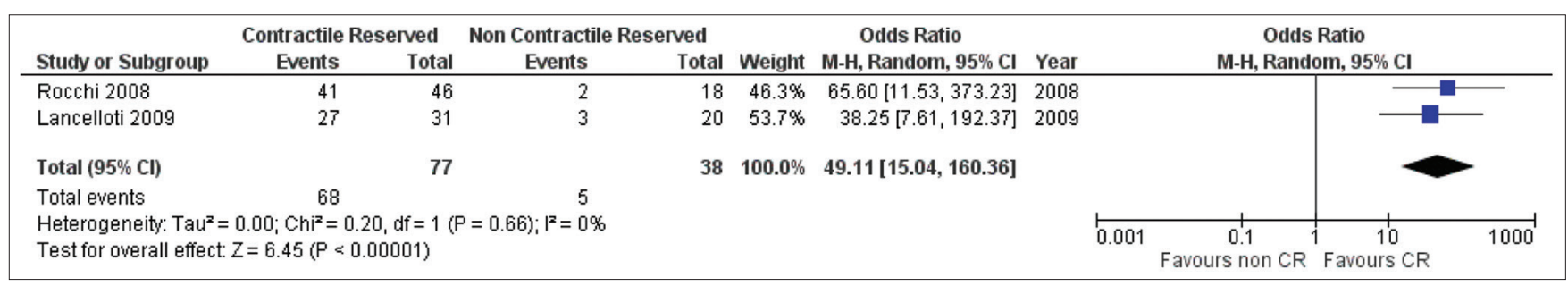

Figure 5: Forest plots of cardiac resynchronization therapy responder based on contractile reserve assessed by exercise stress echocardiography

\begin{tabular}{|c|c|c|c|c|c|c|c|c|c|c|c|}
\hline \multirow[b]{2}{*}{ Study or Subgroup } & \multicolumn{2}{|c|}{ Ischemic } & \multicolumn{2}{|c|}{ Non Ischemic } & \multicolumn{3}{|c|}{ Odds Ratio } & \multirow{2}{*}{\multicolumn{4}{|c|}{$\begin{array}{l}\text { Odds Ratio } \\
\text { M-H, Fixed, } 95 \% \mathrm{Cl}\end{array}$}} \\
\hline & Events & Total & Events & Total & Weight & M-H, Fixed, $95 \% \mathrm{Cl}$ & Year & & & & \\
\hline Da Costa 2006 & 15 & 23 & 32 & 44 & $5.3 \%$ & $0.70[0.24,2.08]$ & 2006 & & & 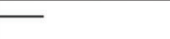 & \\
\hline $\operatorname{Lim} 2007$ & 7 & 9 & 6 & 10 & $0.9 \%$ & $2.33[0.31,17.54]$ & 2007 & & & & \\
\hline Tuccillo 2008 & 7 & 18 & 18 & 24 & $6.5 \%$ & $0.21[0.06,0.80]$ & 2008 & & & & \\
\hline Rocchi 2008 & 9 & 20 & 34 & 44 & $8.0 \%$ & $0.24[0.08,0.74]$ & 2008 & & & & \\
\hline Ciampi 2009 & 21 & 38 & 25 & 31 & $8.5 \%$ & $0.30[0.10,0.89]$ & 2009 & & & & \\
\hline Lancelloti 2009 & 17 & 34 & 13 & 17 & $6.0 \%$ & $0.31[0.08,1.14]$ & 2009 & & & & \\
\hline Senechal 2010 & 20 & 34 & 11 & 15 & $4.3 \%$ & $0.52[0.14,1.97]$ & 2010 & & & & \\
\hline Muto 2010 & 64 & 98 & 106 & 133 & $21.5 \%$ & $0.48[0.27,0.87]$ & 2010 & & & & \\
\hline Chaudhry 2011 & 15 & 32 & 16 & 22 & $6.9 \%$ & $0.33[0.10,1.06]$ & 2011 & & & & \\
\hline Altman 2011 & 4 & 20 & 6 & 11 & $4.3 \%$ & $0.21[0.04,1.05]$ & 2011 & & & & \\
\hline Stankovic 2013 & 13 & 27 & 26 & 31 & $8.6 \%$ & $0.18[0.05,0.60]$ & 2013 & & & & \\
\hline Murin 2014 & 13 & 25 & 15 & 26 & $4.9 \%$ & $0.79[0.26,2.40]$ & 2014 & & & & \\
\hline Mizia-Stec 2014 & 34 & 62 & 48 & 67 & $14.3 \%$ & $0.48[0.23,1.00]$ & 2014 & & & & \\
\hline Total $(95 \% \mathrm{Cl})$ & & 440 & & 475 & $100.0 \%$ & $0.41[0.31,0.55]$ & & & & & \\
\hline Total events & 239 & & 356 & & & & & & & & \\
\hline \multicolumn{8}{|c|}{$\begin{array}{l}\text { Heterogeneity: } \mathrm{Ch}^{2}=10.67, \mathrm{df}=12(\mathrm{P}=0.56) ;\left.\right|^{2}=0 \% \\
\text { Test for overall effect: } Z=6.02(\mathrm{P}=0.00001)\end{array}$} & 0.01 & Non Ischemic & Ischemic & 100 \\
\hline
\end{tabular}

Figure 6: Forest plots benefit of cardiac resynchronization therapy towards ischemic etiology 
a dyssynchrony. Not all ECG with LBBBs reflect a true delay in LV activation. A LV endocardial mapping study has demonstrated that up to one-third of LBBB patients are misdiagnosed [32]. The true LBBB activation induces a peculiar pattern contraction of opposing wall motions (septal flash) with apical rocking movement. Septal flash and apical rocking are two parameters that are widely recommended to assess mechanical dyssynchrony. However, these two parameters have disadvantages, such as; apical rocking sometimes depend on the RV function and there are differences between observers, also there is bias in translation of continuous process to on/off phenomenon in septal flash measurement [31]. Most studies investigated wall movement with echocardiography did not specify the cause of dyssynchronous conditions, weather it was due to electrical activation delayed (broad QRS) or the loading and/or contractile properties. This lack of relation between mechanical and electrical dyssynchrony presumably accounts controversy over echo-dyssynchrony measurement for patient selection and CRT response.

We conducted a systematic review and metaanalysis for the relationship between CR assessed by SE in predicting response to CRT. We found the clinical benefit of SE in CRT responder (OR 7.68; 95\% Cl 4.2713.82; $p<0.001)$. Of the 15 studies assessing CR by DSE, the parameter of reduction in WMSI scores was slightly better than the increase in LVEF (OR 6.86 vs. 5.10) in predicting CRT responder. Contractile reserved assessed by exercise SE showed very high OR (OR 49.11; 95\% Cl 15.04-160.36; p < 0.001). However, only two studies evaluated contractile reserved assessed by exercise SE, more study in needed to draw a conclusion that exercise is better than DSE in predicting CRT responder.

Our results are consistent with previous studies and the recommendations of the European Association of Cardiovascular Imaging and ASE that clinical assessment of SE can be performed beyond coronary artery disease [8], [9], [32]. The absence of CR is a strong determinant of outcome and a potential marker of negative response to CRT [33]. Actually, no reliably echocardiographic dyssynchrony measurements have been shown to predict the CRT responder. Several studies even suggest that the CR tends to be a stronger response predictor and offers incremental value above dyssynchrony measurements [9], [21]. This result also shifts the topic of functional response to the myocardial substrate from mechanical and electrical dyssynchrony. It is possible that similar information can be obtained with other techniques myocardial viability testing with metabolic, structural, or functional markers, such as delayed enhancement, nuclear imaging, or low-dose dobutamine magnetic resonance [8].

CR appears to be present independently of HF etiology. Previous meta-analysis found that there were no significant differences in the proportion of patients with ischemic and non-ischemic cardiomyopathy [9]. From our meta-analysis, we found that patient with nonischemic etiology has better respond to CRT compare to patient with ischemic etiology (OR 0.41; 95\% Cl $0.31-0.55, p<0.01)$. This indicates the myocardium itself has a lack of contractility due to metabolic and/or ultrastructural changes in ischemic patient. In any event, monitoring of the CR tends to be an essential modality for ensuring the response in patients expected to have a high response rate of non-ischemic etiology [9].

Limitations of this meta-analysis were the substantial heterogeneity between studies. This heterogeneity could be due to different SE administration (exercise and dobutamine), different CR parameters, and different CRT responder criteria. For the SE group with dobutamine, heterogeneity could be caused by different dobutamine doses, ranging from 10 to $40 \mathrm{mcg}$. For the same stress at the same dose, the CR parameters differed across studies (EF and WMSI), and when the parameters were the same, varied cutoffs were used in each studies (Table 1). Heterogeneity was substantially decreased in the dobutamine study with WMSI parameter $\left(I^{2}=21 \%\right)$ compared with $E F$ $\left(I^{2}=77 \%\right)$ and overall analysis $\left(I^{2}=68 \%\right)$.

\section{Conclusion}

Our meta-analysis shows that the presence of CR assessed by both dobutamine and exercise SE is associated with a better positive CRT response. These results can serve as the basis for updating the guidelines for the management of severe heart failure, which currently recommends clinical symptoms, ejection fraction, and QRS complex duration to identify CRT candidates.

\section{References}

1. Lozano I, Bocchiardo M, Achtelik M, Gaita F, Trappe H, Daoud E et al. Impact of biventricular pacing on mortality in a randomized crossover study of patients with heart failure and ventricular arrhythmias. Pacing Clin Electrophysiol. 2000;23(11 Pt 2):1711 2. https://doi.org/10.1111/j.1540-8159.2000.tb07001.x PMid:11139906

2. Abraham WT, Hayes DL. Cardiac resynchronization therapy for heart failure. Circulation. 2003;108(21):2596-603. https://doi. org/10.1161/01.cir.0000096580.26969.9a PMid:14638522

3. Ponikowski $P$, Voors AA, Anker SD, Bueno H, Cleland JG, Coats AJ, et al. 2016 ESC Guidelines for the diagnosis and treatment of acute and chronic heart failure. Eur Heart J. 2016;37(27):2129-200. https://doi.org/10.1093/eurheartj/ehw128 PMid:27206819 
4. Pitzalis MV, lacoviello M, Romito R, Massari F, Rizzon B, Luzzi G, et al. Cardiac resynchronization therapy tailored by echocardiographic evaluation of ventricular asynchrony. J Am Coll Cardiol. 2002;40(9):1615-22. https://doi.org/10.1016/ s0735-1097(02)02337-9

\section{PMid:12427414}

5. Hummel JP, Lindner JR, Belcik JT, Ferguson JD, Mangrum JM, Bergin JD, et al. Extent of myocardial viability predicts response to biventricular pacing in ischemic cardiomyopathy. Hear Rhythm. 2005;2(11):1211-7. https://doi.org/10.1016/j. hrthm.2005.07.027

PMid:16253911

6. Kawara T, Derksen R, de Groot JR, Coronel R, Tasseron S, Linnenbank AC, et al. Activation delay after premature stimulation in chronically diseased human myocardium relates to the architecture of interstitial fibrosis. Circulation. 2001;104(25):3069-75. https://doi.org/10.1161/hc5001.100833 PMid:11748102

7. Bax JJ, Wijns W, Cornel JH, Visser FC, Boersma E, Fioretti PM. Accuracy of currently available techniques for prediction of functional recovery after revascularization in patients with left ventricular dysfunction due to chronic coronary artery disease: Comparison of pooled data. J Am Coll Cardiol. 1997;30(6):145160. https://doi.org/10.1016/s0735-1097(97)00352-5

PMid:9362401

8. Ciampi Q, Carpeggiani C, Michelassi C, Villari B, Picano E. Left ventricular contractile reserve by stress echocardiography as a predictor of response to cardiac resynchronization therapy in heart failure: A systematic review and meta-analysis. BMC Cardiovasc Disord. 2017;17(1):223. https://doi.org/10.1186/ s12872-017-0657-4

PMid:28814264

9. Kloosterman M, Damman K, Van Veldhuisen DJ, Rienstra M Maass AH. The importance of myocardial contractile reserve in predicting response to cardiac resynchronization therapy. Eur J Heart Fail. 2017;19(7):862-9. https://doi.org/10.1002/ejhf.768 PMid:28233406

10. Hutton B, Salanti G, Caldwell DM, Chaimani A, Schmid CH, Cameron $\mathrm{C}$, et al. The PRISMA extension statement for reporting of systematic reviews incorporating network meta-analyses of health care interventions: Checklist and explanations. Ann Intern Med. 2015;162(11):777-84. https://doi.org/10.7326/m14-2385 PMid:26030634

11. Wells G, Shea B, O'Connell D, Peterson J, Welch V, Losos M, et al. The Newcastle-Ottawa Scale (NOS) for Assessing the Quality of Nonrandomised Studies in Meta-analyses; 2013.

12. Da Costa A, Thévenin J, Roche F, Faure E, RoméyerBouchard C, Messier M, et al. Prospective validation of stress echocardiography as an identifier of cardiac resynchronization therapy responders. Hear Rhythm. 2006;3(4):406-13. https:// doi.org/10.1016/j.hrthm.2005.12.017

PMid:16567286

13. Ypenburg C, Sieders A, Bleeker GB, Holman ER, van der Wall EE, Schalij MJ, et al. Myocardial contractile reserve predicts improvement in left ventricular function after cardiac resynchronization therapy. Am Heart J. 2007;154(6):1160-5. https://doi.org/10.1016/j.ahj.2007.07.035 PMid: 18035090

14. Lim P, Bars C, Mitchell-Heggs L, Roiron C, Elbaz N, Hamdaoui B, et al. Importance of contractile reserve for CRT. Europace. 2007;9(9):739-43. https://doi.org/10.1093/europace/eum117 PMid: 17573360

15. Tuccillo B, Muto C, lengo R, Accadia M, Rumolo S, Canciello M, et al. Presence of left ventricular contractile reserve, evaluated by means of dobutamine stress-echo test, is able to predict response to cardiac resynchronization therapy. J Interv Card Electrophysiol. 2008;23(2):121-6. https://doi.org/10.1007/ s10840-008-9255-9

PMid: 18587637

16. Rocchi G, Bertini M, Biffi M, Ziacchi M, Biagini E, Gallelli I, et al. Exercise stress echocardiography is superior to rest echocardiography in predicting left ventricular reverse remodelling and functional improvement after cardiac resynchronization therapy. Eur Heart J. 2008;30(1):89-97. https://doi.org/10.1093/eurheartj/ehn483

PMid:18987095

17. Lancellotti P, Senechal M, Moonen M, Donal E, Magne J, Nellessen E, et al. Myocardial contractile reserve during exercise predicts left ventricular reverse remodelling after cardiac resynchronization therapy. Eur J Echocardiogr. 2009;10(5):6638. https://doi.org/10.1093/ejechocard/jep033

PMid:19351652

18. Ciampi Q, Pratali L, Citro R, Piacenti M, Villari B, Picano E. Identification of responders to cardiac resynchronization therapy by contractile reserve during stress echocardiography. Eur J Heart Fail. 2009;11(5):489-96. https://doi.org/10.1093/ eurjhf/hfp039 PMid:19324921

19. Sénéchal M, Lancellotti P, Magne J, Garceau P, Champagne J, Blier $\mathrm{L}$, et al. Contractile reserve assessed using dobutamine echocardiography predicts left ventricular reverse remodeling after cardiac resynchronization therapy: Prospective validation in patients with left ventricular dyssynchrony. Echocardiography. 2010;27(6):668-76. https://doi org/10.1111/j.1540-8175.2009.01106.x

PMid:20345438

20. Muto C, Gasparini M, Neja CP, lacopino S, Davinelli M, Zanon F, et al. Presence of left ventricular contractile reserve predicts midterm response to cardiac resynchronization therapyresults from the LOw dose DObutamine Stress-Echo test in cardiac resynchronization therapy (LODO-CRT) trial. Hear Rhythm. 2010;7(11):1600-5. https://doi.org/10.1016/j.hrthm.2010.07.036 PMid:20691283

21. Chaudhry FA, Shah A, Bangalore S, Derose J, Steinberg JS Inotropic contractile reserve and response to cardiac resynchronization therapy in patients with markedly remodeled left ventricle. J Am Soc Echocardiogr. 2011;24(1):91-7. https:// doi.org/10.1016/j.echo.2010.10.007 PMid:21126856

22. Altman RK, McCarty D, Chen-Tournoux AA, Tournoux FB Riedl L, Orencole M, et al. Usefulness of low-dose dobutamine echocardiography to predict response and outcome in patients undergoing cardiac resynchronization therapy. Am J Cardiol. 2011;108(2):252-7. https://doi.org/10.1016/j. amjcard.2011.03.033

PMid:21550579

23. Gasparini M, Muto C, lacopino S, Zanon F, Dicandia C, Distefano G, et al. Low-dose dobutamine test associated with interventricular dyssynchrony: A useful tool to identify cardiac resynchronization therapy responders: Data from the LOw dose DObutamine stress-echo test in Cardiac Resynchronization Therapy (LODO-CRT) phase 2 study. Am Heart J. 2011;163(3):422-9. https://doi.org/10.1016/j.ahj.2011.11.015 PMid:22424013

24. Pugllese M, Minardi G, Vitali A, Natale E, de Girolamo P, Zampi G, et al. Influence of myocardial viability on responsiveness to cardiac resynchronization in ischemic dilated cardiomyopathy: $A$ prospective observational cohort study. Anadolu Kardiyol Derg. 2012;12(2):132-41. https://doi.org/10.5152/akd.2012.039 PMid:22281793

25. Stankovic I, Aarones M, Smith HJ, Vörös G, Kongsgaard E, 
Neskovic AN, et al. Dynamic relationship of left-ventricular dyssynchrony and contractile reserve in patients undergoing cardiac resynchronization therapy. Eur Heart J. 2013;35(1):4855. https://doi.org/10.1093/eurheartj/eht294 PMid:23918757

26. Mizia-Stec K, Wita K, Mizia M, Szwed H, NowalanyKozielska E, Chrzanowski $\measuredangle$, et al. Preserved contractile reserve in a dobutamine test for the prediction of a response to resynchronisation therapy in ischemic and non-ischemic cardiomyopathy a multicenter ViaCRT study. Int J Cardiol. 2014;172(2):476-7. https://doi.org/10.1016/j.jicard.2013.12.204 PMid:24456874

27. Murín P, Mitro P, Valocik G, Spurný P. Global myocardial contractile reserve assessed by high-dose dobutamine stress echocardiography predicts response to the cardiac resynchronization therapy. Echocardiography. 2014;32(3):4905. https://doi.org/10.1111/echo.12694

\section{PMid:25059770}

28. Płońska-Gościniak E, Kasprzak JD, Kukulski T, Mizia-Stec K, Nowalany-Kozielska E, Gąsior Z, et al. The role of low-dose dobutamine echocardiography in predicting response to biventricular pacing: Results from the multicentre viability in cardiac resynchronisation therapy (ViaCRT) study. Polish Arch Intern Med. 2016;126(12):989-94. https://doi.org/10.20452/ pamw.3715

PMid:27958262

29. Young JB. Combined cardiac resynchronization and implantable cardioversion defibrillation in advanced chronic heart failure. JAMA. 2003;289(20):2685. https://doi.org/10.1001/ jama.289.20.2685

PMid: 12771115

30. Cleland JG, Daubert JC, Erdmann E, Freemantle N, Gras D, Kappenberger $\mathrm{L}$, et al. The effect of cardiac resynchronization on morbidity and mortality in heart failure. $\mathrm{N}$ Engl $\mathrm{J}$ Med. 2005;352(15):1539-49. https://doi.org/10.1056/nejmoa050496 PMid: 15753115

31. Spartalis M, Tzatzaki E, Spartalis E, Damaskos C, Athanasiou A, Livanis $\mathrm{E}$, et al. The role of echocardiography in the optimization of cardiac resynchronization therapy: Current evidence and future perspectives. Open Cardiovasc Med J. 2017;11(1):13345. https://doi.org/10.2174/1874192401711010133 PMid:29387277

32. Papageorgiou N, Providência R, Lambiase PD, Tousoulis D, Lloyd G, Bhattacharyya S. Does presence of left ventricular contractile reserve improve response to cardiac resynchronization therapy? An updated meta-analysis. Int J Cardiol. 2018;252:224-8. https://doi.org/10.1016/j. ijcard.2017.09.034 PMid:29249433

33. Lancellotti P, Pellikka PA, Budts W, Chaudhry FA, Donal E, Dulgheru $\mathrm{R}$, et al. The clinical use of stress echocardiography in non-ischemic heart disease: Recommendations from the European association of cardiovascular imaging and the American society of echocardiography. J Am Soc Echocardiogr. 2017;30(2):101-38. https://doi.org/10.1016/j.echo.2016.10.016 PMid:28164802 